\title{
A temperature-sensitive phase-change hydrogel of tamoxifen achieves the long-acting antitumor activation on breast cancer cells
}

This article was published in the following Dove Press journal: OncoTargets and Therapy

\author{
Du Meng ${ }^{1, *}$ \\ Hongwei Lei ${ }^{2} *$ \\ Xiaoqiang Zheng ${ }^{3}$ \\ Yaxuan $\mathrm{Han}^{4}$ \\ Ronggang Sun ${ }^{5}$ \\ Dongli Zhaol \\ Rui Liu' \\ 'Department of Radio Oncology, The \\ First Affiliated Hospital of Xi'an Jiaotong \\ University School of Medicine, Xi'an, \\ Shaanxi Province, 71006I, People's \\ Republic of China; ${ }^{2}$ Department of Radio \\ Oncology, The Second Affiliated Hospital \\ of Dalian Medical University, Dalian, \\ Liaoning Province, I 16027, People's \\ Republic of China; ${ }^{3}$ Department of \\ Medical Oncology, The First Affiliated \\ Hospital of Xi'an Jiaotong University \\ School of Medicine, Xi'an, Shaanxi \\ Province, 71006I, People's Republic of \\ China; ${ }^{4}$ Department of Oncology, The \\ Xi'an Chest Hospital, Xi'an, Shaanxi \\ Province, 710000, People's Republic of \\ China; ${ }^{5}$ Department of Radio Oncology, \\ The People's Hospital of YangZhong City, \\ YangZhong, Jiangsu Province, 212200, \\ People's Republic of China
}

*These authors contributed equally to this work

Correspondence: Rui Liu

Department of Radio Oncology, The First Affiliated Hospital of Xi'an Jiaotong University School of Medicine, Xi'an 71006I, People's Republic of China

Tel +862985324029

Fax +86 2985324029

Email reon10@sohu.com
Background: Breast cancer is one of the foremost threats to female health nowadays Tamoxifen, an antagonist of estrogen receptor- $\alpha(E R \alpha)$, is the first choice for endocrinedependent breast cancer (ER $\alpha$-positive breast cancer) treatment. However, ER $\alpha$ has an important function in the normal physical regulation of estrogen, and current oral administration of tamoxifen has potential side effects on normal endocrine secretion. In the present work, we aim to develop novel approaches to increase the antitumor effect of tamoxifen on breast cancer cells and decrease the potential side effects in the human body during treatment.

Methods: A temperature-sensitive phase-change hydrogel for tamoxifen (Tam-Gel) was generated. After establishing subcutaneous tumors formed by MCF-7, an ER $\alpha$-positive breast cancer cell line, in nude mice, an intratumoral injection of Tam-Gel was performed to examine whether Tam-Gel facilitated the slow-release or antitumor effect of tamoxifen. A metastatic breast cancer model was established using the intrahepatic growth of MCF-7 cells in immunodeficient rats.

Results: Tam-Gel can transform from liquid to hydrogel at room temperature. An intratumoral injection of Tam-Gel facilitated the slow-release or antitumor effect of tamoxifen. Once Tam-Gel, but not Tam-Sol, was administered by intratumoral injection, it significantly decreased the uptake of radionuclide probes $\left({ }^{18} \mathrm{~F}\right.$-fluoroestradiol or ${ }^{18} \mathrm{~F}$-fluorodeoxyglucose $)$ by cells in rats' livers and the intrahepatic growth of MCF-7 cells in rats' livers.

Conclusion: A novel slow-release system was successfully prepared to facilitate the longterm release of tamoxifen in breast cancer tissues, and achieved an antitumor effect in the long term.

Keywords: breast cancer, estrogen receptor- $\alpha$, tamoxifen nanoparticle, temperature-sensitive phase-change hydrogel

\section{Introduction}

Breast cancer is one of the foremost threats to female health nowadays. ${ }^{1-3}$ Among the pathological types, endocrine-dependent breast cancer (estrogen receptor- $\alpha$ $[\mathrm{ER} \alpha]$-positive breast cancer) is the most common subtype of breast cancer and accounts for most cases. Thus, ER $\alpha$ has been confirmed to be the primary target for endocrine-dependent breast cancer. ${ }^{4-7}$ Tamoxifen, an oral antagonist of ER $\alpha$, is the first choice for ER $\alpha$-positive breast cancer treatment. ${ }^{8-11}$ Although the application of an oral antagonist of ER $\alpha$ could significantly prolong survival and improve the quality of life of patients with breast cancer, there are still some problems: 1) ER $\alpha$ is not only a therapeutic target of endocrine-dependent breast cancer but also an 
important regulator of human physical processes, and oral administration of tamoxifen has potential side effects on cardiovascular and muscular systems; ${ }^{12-15}$ and 2) administration of tamoxifen orally or by an intravenous drip will enable the systemic distribution of tamoxifen in the body and thus limit the concentration of the drug in the breast cancer lesion. ${ }^{16-20}$ Therefore, it is valuable to develop novel approaches to increase the safety and efficacy of an oral antagonist of ER $\alpha$ for endocrine-dependent breast cancer treatment.

Although the existing antitumor treatment strategy is based on oral or intravenous drip therapy strategies, the precise administration of antitumor drugs in localized tumor tissues has been achieved by several image-guided interventional techniques to avoid various toxic and side effects caused by antitumor drugs. ${ }^{21-23}$ Therefore, preparing slow-release and long-acting formulations of antitumor drugs can not only achieve long-term antitumor effects by single administration but also help to avoid the side effects of the drug. ${ }^{24,25}$ In the present work, a temperature-sensitive phase-change hydrogel of tamoxifen was established. Tamhydrogel (Tam-Gel) was injected intratumorally in established subcutaneous MCF-7 tumors or intrahepatic MCF-7 tumors. The slow release of tamoxifen in the blood and tumor tissues was measured. Tumor growth was identified by measuring tumor volumes, tumor weight and nodule area, or by micro-positron emission tomography (microPET) screening.

\section{Material and methods}

\section{Cell lines and agents}

MCF-7 cells (an ER $\alpha$-positive breast cancer cell line) were purchased from the Type Culture Collection of the Chinese Academy of Sciences (Shanghai, People's Republic of China), an organization possessing biological samples typical to the Chinese government, a gift from Dr Feng Fan maintained under conditions described in the previous work. $^{26}$ Tamoxifen (cat no S1192) was purchased from the Selleck Corporation (Houston, TX, USA). Poly(lactic-coglycolic acid)- polyethylene glycol (PLGA-PEG) was purchased from Dai-Gang Corporation (Jinan City, Shandong Province, People's Republic of China). The results of infrared detection and nuclear magnetic detection at similar physiological temperatures of PLGA-PEG are shown in Figures S1 and S2. All experiments, protocols and use of cell lines were approved by the Ethics Committee of the First Affiliated Hospital of Xi'an Jiao Tong University.

\section{Preparation of tamoxifen formulations}

To prepare tamoxifen-hydrogel (Tam-Gel), tamoxifen citrate powder was accurately dissolved in acetone, and the prepared acetone solution was slowly added dropwise to a $5 \%$ aqueous solution of glucose, under stirring, to obtain a suspension of tamoxifen nanoparticles (nanosuspension). The amount of acetone used in preparing tamoxifen was $1 \mathrm{~mL}$ of acetone per $1 \mathrm{mg}$ of drug. Acetone was removed from nanosuspension using a dialysis method, and the suspension was freeze-dried to remove water, resulting in nanoparticle freeze-dried powder. The solution of PLGA-PEG-PLGA in PBS was prepared at $10^{\circ} \mathrm{C}$ and added to the nanoparticle freeze-dried powder. The mixture was stirred at $10^{\circ} \mathrm{C}$ to prepare the tamoxifen hydrogel (named Tam-Gel). Acetone was removed from the suspension of tamoxifen nanoparticles (nanosuspension) in PBS; this was named Tam-Sol and used as a control. The final concentration of tamoxifen in its Tam-Sol or Tam-Gel form was almost $2 \mathrm{mg} / \mathrm{mL}$. To examine the phase transition of Tam-Gel from liquid to hydrogel, the apparent viscosity $\eta(\mathrm{Pa} / \mathrm{s})$ of Tam-Gel with different concentrations of PLGA-PEG-PLGA as a function of temperature $\left({ }^{\circ} \mathrm{C}\right)$ was examined. The phase-transition temperature $\left(T_{1 / 2}\right)$ was calculated based on apparent viscosity-temperature curves. The phase transition of hydrogels with indicated PLGA-PEG-PLGA amounts (5.0, 7.5, 10.0, 12.5 and $15.0 \%$ ) was examined. The effect of $\mathrm{pH}$ values on the phase transition of hydrogels was also examined under indicated $\mathrm{pH}$ values (7.4, 7.2, 7.0, 6.8 and 6.6).

After the tamoxifen had been dissolved in an organic solvent (eg, DMSO), the tamoxifen solution was obtained by dilution with PBS to form the Tam-Sol formulation, used as a control. The concentrations of tamoxifen in TamGel and Tam-Sol were basically the same, by controlling the amount of PBS added to the formulations.

\section{In vitro and in vivo release of tamoxifen from Tam-Gel}

For the in vitro release of tamoxifen from Tam-Gel, Tam-Gel was incubated in sterilized PBS. Samples of PBS were collected at indicated time-points and extracted with acetonitrile (ACN). Next, the tamoxifen in the samples was detected by liquid chromatography-tandem mass spectrometry (LC-MS /MS), as described in previous publications. ${ }^{27,28}$

For the in vivo release of tamoxifen from Tam-Gel, subcutaneous tumor animal models were used. All animal experiments included in the present work were permitted by 
the Institutional Animal Care and Use Committee of The First Affiliated Hospital of Xi' an Jiaotong University School of Medicine and were carried out in accordance with the UK Animals (Scientific Procedures) Act of 1986 and associated guidelines. MCF-7 cells were injected into nude mice $\left(1 \times 10^{7}\right.$ cells per animal) to form subcutaneous tumors. ${ }^{29,30}$ The tumor volumes were calculated as: Tumor length $\times$ Tumor width $\times$ Tumor width $/ 2$. When the volume of the subcutaneous tumors reached almost $1200-1500 \mathrm{~mm}^{3}$, the tamoxifen formulations (Tam-Sol and Tam-Gel) were injected into these tumors. Nude mice were harvested at indicated timepoints, and tumor tissues were collected. Collected tumor tissues were lysed, and the tamoxifen remaining in the tumor tissues was extracted with ACN for LC-MS/MS examination following the methods described previously. ${ }^{27,28}$

\section{In vivo antitumor effects of tamoxifen formulations}

For the subcutaneous tumor model, MCF-7 cells were injected into nude mice. When tumor volumes reached almost 1000-1200 $\mathrm{mm}^{3}$, Tam-Sol or Tam-Gel was injected into the tumor tissues. Each tumor could be injected with about $50 \mu \mathrm{L}$ of tamoxifen formulations. After 3-4 weeks' growth, tumors were harvested, and the tumor weights or volumes were measured.

For the intrahepatic model in immunodeficient rats, MCF-7 cells were first seeded into nude mice to form subcutaneous tumors. Next, subcutaneous tumor tissues formed by MCF-7 cells were harvested and microblocks of subcutaneous tumor tissues with equal weights were prepared via weighing using a precision balance. Then, microblocks of subcutaneous tumor tissues with equal weights were seeded into immunodeficient rats' livers by open laparotomy (Table S1). After 5-6 weeks' growth, intrahepatic tumors formed in the rats' livers. Formulations of tamoxifen, Tam-Sol or Tam-Gel were injected into the MCF-7 lesions in the rats' livers by open laparotomy (50 $\mu \mathrm{L}$ per injection). At each time-point, mice were injected intravenously with $200 \mu \mathrm{Ci}(7.4 \mathrm{MBq})$ of ${ }^{18} \mathrm{~F}$-radiolabeled fluorodeoxyglucose $\left({ }^{18} \mathrm{~F}\right.$-FDG) or $100 \mu \mathrm{Ci}(3.7 \mathrm{MBq})$ of ${ }^{18} \mathrm{~F}$-radiolabeled fluoroestradiol $\left({ }^{18} \mathrm{~F}\right.$-FES) and examined by a positron emission tomography/computed tomography (PET/ CT) scanner (Philips, Amsterdam, The Netherlands) following previously published methods. ${ }^{31-33} \mathrm{Ci}$ is a unit of nuclear intensity: 1 Curie $(\mathrm{Ci})$ equals $3.7 \times 10^{10}$ Becquerels $(\mathrm{Bq})$; so, in this study, $200 \mu \mathrm{Ci}=7.4 \mathrm{MBq}$ and $100 \mu \mathrm{Ci}=3.7 \mathrm{MBq}$. Results from images or liver organs were quantifiably analyzed by ImageJ software following previous methods. ${ }^{34,35}$

\section{Ethics statement}

The use of cell lines was approved by the Ethics Committee of The First Affiliated Hospital of Xi'an Jiaotong University School of Medicine. The use of animals was permitted by the Institutional Animal Care and Use Committee of The First Affiliated Hospital of Xi'an Jiaotong University School of Medicine and were carried out in accordance with the UK Animals (Scientific Procedures) Act of 1986 and associated guidelines.

\section{Statistical analysis}

Statistical analysis was carried out by a two-way ANOVA without Bonferroni correction using SPSS statistics software (IBM Corp., Armonk, NY, USA). The phase-transition temperature $\left(T_{1 / 2}\right)$ of Tam-Gel or half-life ( $t_{1 / 2}$ value) of tamoxifen in Tam-Gel or the tumor tissues was calculated with Origin statistics software (version 8.5; OriginLab Corp., Northampton, MA, USA). A $P$-value $<0.05$ was considered to indicate a statistically significant difference between groups.

\section{Results \\ Preparation of the temperature-sensitive phase-transition hydrogel of tamoxifen}

First, the temperature-sensitive phase transition of tamoxifen was prepared, and its physicochemical properties were identified. Table 1 shows the $T_{1 / 2}$ values of Tam-Gel containing the indicated concentrations of PLGA-PEG-PLGA. The $T_{1 / 2}$ value of Tam-Gel containing 10\% PLGA-PEG-PLGA was nearest to body temperature $\left(37.14 \pm 0.66^{\circ} \mathrm{C}\right)$, so the $10 \%$ concentration of PLGA-PEG-PLGA was used in the next steps of the experiments. Table 2 shows the $T_{1 / 2}$ values of Tam-Gel containing 10\% PLGA-PEG-PLGA at the indicated $\mathrm{pH}$ values. The results show that the changes in $\mathrm{pH}$ did not affect the $T_{1 / 2}$ of hydrogel within the selected $\mathrm{pH}$ range.

Next, Tam-Gel images from the transmission electron microscope were obtained. As shown in Figure 1, the particle diameter of the tamoxifen nanoparticles was

Table I Phase-transition $\left(T_{1 / 2}\right)$ values of Tam-Gel at indicated concentrations of PLGA-PEG-PLGA

\begin{tabular}{|l|l|}
\hline PLGA-PEG-PLGA (\%) & $\boldsymbol{T}_{\mathbf{1 / 2}}\left({ }^{\circ} \mathbf{C}\right)$ \\
\hline 5.0 & $53.38 \pm 0.17$ \\
7.5 & $44.92 \pm 0.53$ \\
10.0 & $37.14 \pm 0.66$ \\
12.5 & $27.69 \pm 0.44$ \\
15.0 & $15.08 \pm 0.11$ \\
\hline
\end{tabular}


Table 2 Phase-transition $\left(T_{1 / 2}\right)$ values of Tam-Gel at indicated $\mathrm{pH}$ values

\begin{tabular}{|l|l|}
\hline $\mathbf{p H}$ & $\boldsymbol{T}_{\mathbf{1 / 2}}\left({ }^{\circ} \mathbf{C}\right)$ \\
\hline 7.4 & $36.82 \pm 0.5 \mathrm{I}$ \\
7.2 & $37.08 \pm 0.22$ \\
7.0 & $37.17 \pm 1.35$ \\
6.8 & $37.48 \pm 0.84$ \\
6.6 & $37.89 \pm 0.74$ \\
\hline
\end{tabular}

almost $120 \mathrm{~nm}$ and the particles were uniformly distributed in the PLGA-PEG-PLGA hydrogel. Therefore, we successfully prepared a tamoxifen temperature-sensitive phase-change hydrogel (Tam-Gel).

\section{In vitro release of tamoxifen from Tam-Gel}

To examine whether Tam-Gel could achieve the slow release of tamoxifen, the in vitro release of tamoxifen from Tam-Gel to
PBS was examined by LC-MS/MS experiments. As shown in Figure 2, Tam-Gel achieved the slow release of tamoxifen from the temperature-sensitive phase-transition hydrogel of tamoxifen (Tam-Gel). Tamoxifen was sustained in Tam-Gel for more than 400 hours and the half-life $\left(t_{1 / 2}\right)$ of tamoxifen release from Tam-Gel (sustained in hydrogel) was 194.41 \pm 12.60 hours. Table 3 shows the $t_{1 / 2}$ values of tamoxifen release from Tam-Gel at the indicated $\mathrm{pH}$ values. The results indicate that the changes in $\mathrm{pH}$ did not affect the $t_{1 / 2}$ of tamoxifen release from Tam-Gel within the selected $\mathrm{pH}$ range ( $\mathrm{pH}$ 7.4-6.6). Therefore, the preparation of tamoxifen in the Tam-Gel formulation achieved the slow release of in vitro tamoxifen.

\section{In vivo release of tamoxifen from tumor tissues injected with tamoxifen formulations}

To examine whether the Tam-Gel preparation could achieve the in vivo slow release or long-term sustained
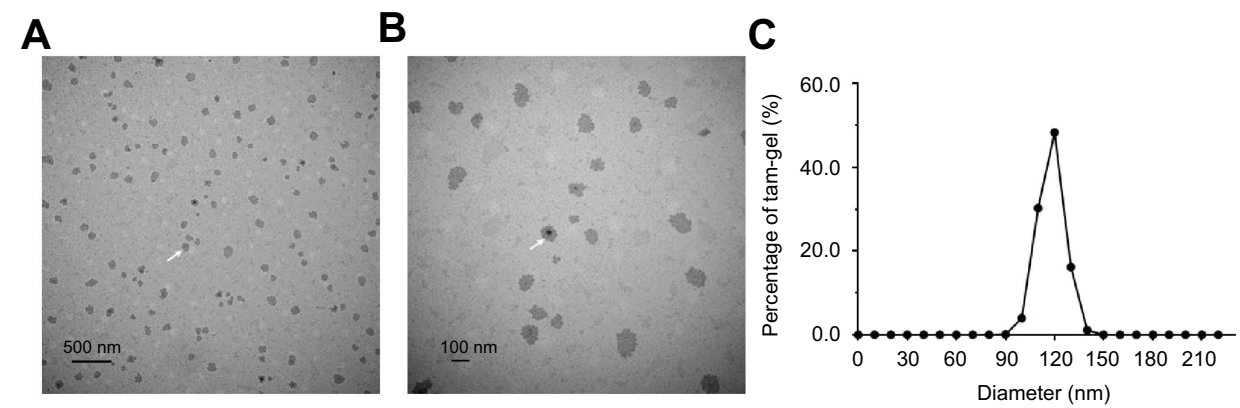

Figure I Characteristics of temperature-sensitive phase-change hydrogels of tamoxifen. (A, B) images of Tam-Gel from the transmission electron microscope. The scale bar indicates $500 \mathrm{~nm}$ (A) or $100 \mathrm{~nm}$ (B). (C) Particle diameters of tamoxifen nanoparticles, shown as a distribution figure. White arrows indicate the tamoxifen nanoparticles (A, B).
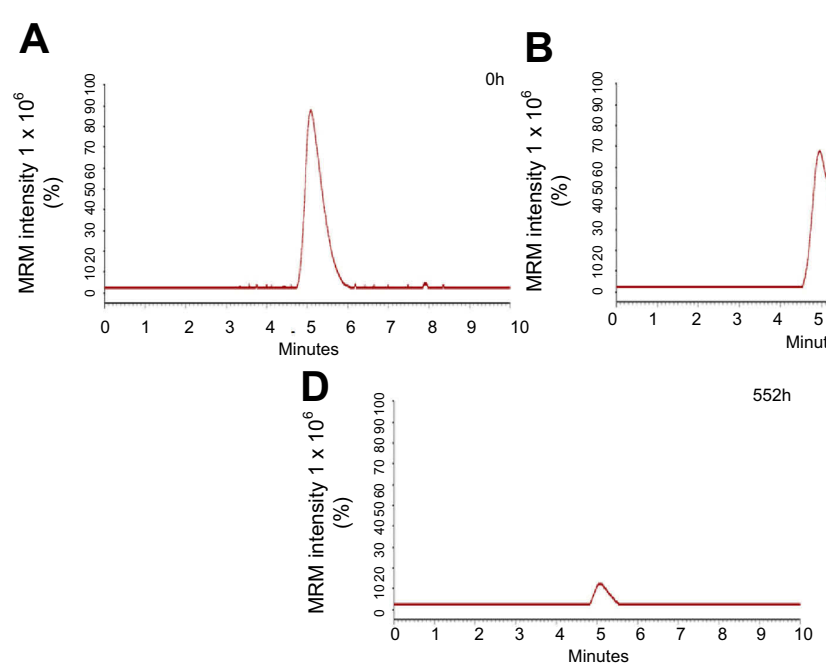
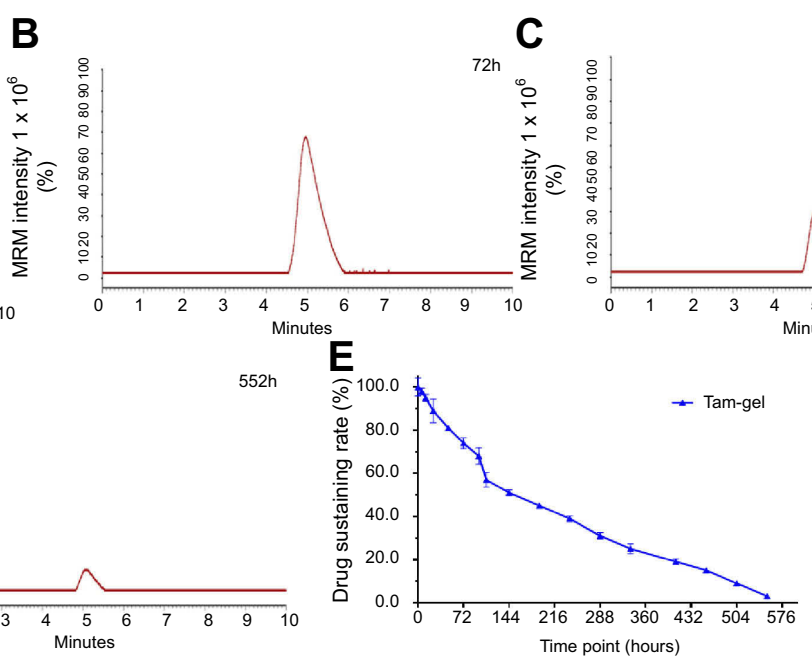

Figure 2 Preparation of Tam-Gel achieves in vitro long-term release of tamoxifen from Tam-Gel. Tam-Gel was incubated with PBS. Then, tamoxifen sustained in Tam-Gel samples at the indicated time-points was determined by LC-MS/MS experiments. Representative LC-MS/MS maps are shown at (A) 0 hours, (B) 72 hours, (C) 408 hours, and (D) 522 hours. (E) Sustained release curve of tamoxifen in Tam-Gel (mean \pm SD).

Abbreviation: LC-MS/MS, liquid chromatography-tandem mass spectrometry. 
Table 3 Half-life $\left(t_{1 / 2}\right)$ times of tamoxifen release from Tam-Gel at indicated $\mathrm{pH}$ values

\begin{tabular}{|l|l|}
\hline $\mathbf{p H}$ & $\boldsymbol{t}_{\mathbf{1 / 2}}$ (hours) \\
\hline 7.4 & $201.91 \pm 7.25$ \\
7.2 & $198.06 \pm 14.52$ \\
7.0 & $195.12 \pm 9.88$ \\
6.8 & $190.50 \pm 3.50$ \\
6.6 & $188.55 \pm 11.03$ \\
\hline
\end{tabular}

features of tamoxifen in tumor tissues, MCF-7 cells were subcutaneously injected into nude mice to form subcutaneous tumors. Once the volumes of the subcutaneous tumors had reached $1200-1500 \mathrm{~mm}^{3}$, tamoxifen formulations (Tam-Gel or Tam-Sol) were injected into the subcutaneous tumors, and the tumor tissues were harvested at the indicated time-points for LC-MS/MS analysis. As shown in Figure 3, after intratumoral injection of Tam-Sol, tamoxifen was quickly released and almost completely cleared from the tumor tissues within 48 hours. The $t_{1 / 2}$ value of tamoxifen in tumor tissues injected with tamoxifen (Tam-Sol) was $13.08 \pm 1.14$ hours.

Moreover, the intratumoral injection of Tam-Gel achieved slower and more sustained release of tamoxifen in tumor tissues compared to the Tam-Sol injection group. The $t_{1 / 2}$ value of tamoxifen in tumor tissues injected with tamoxifen (Tam-Gel) was $137.95 \pm 11.72$ hours. Moreover, after the injection of Tam-Gel in the
A
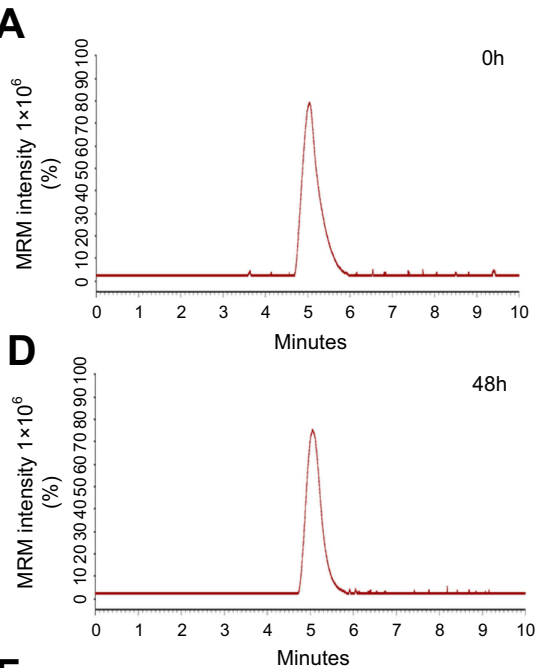

$\mathbf{F}$
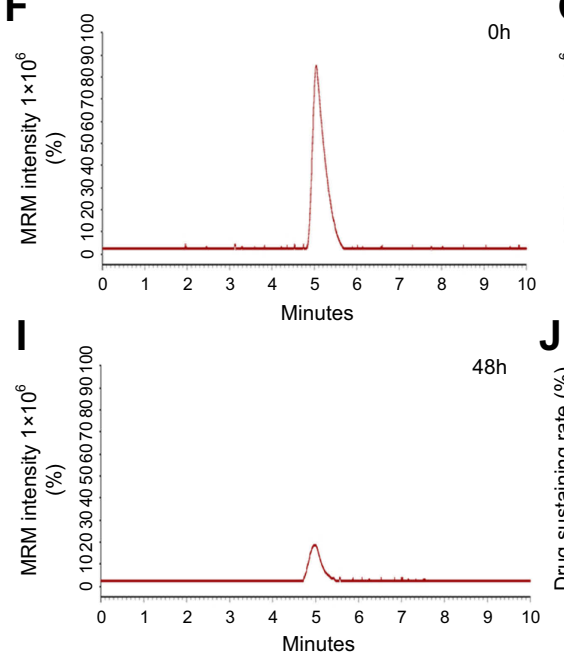

$\mathbf{B}$
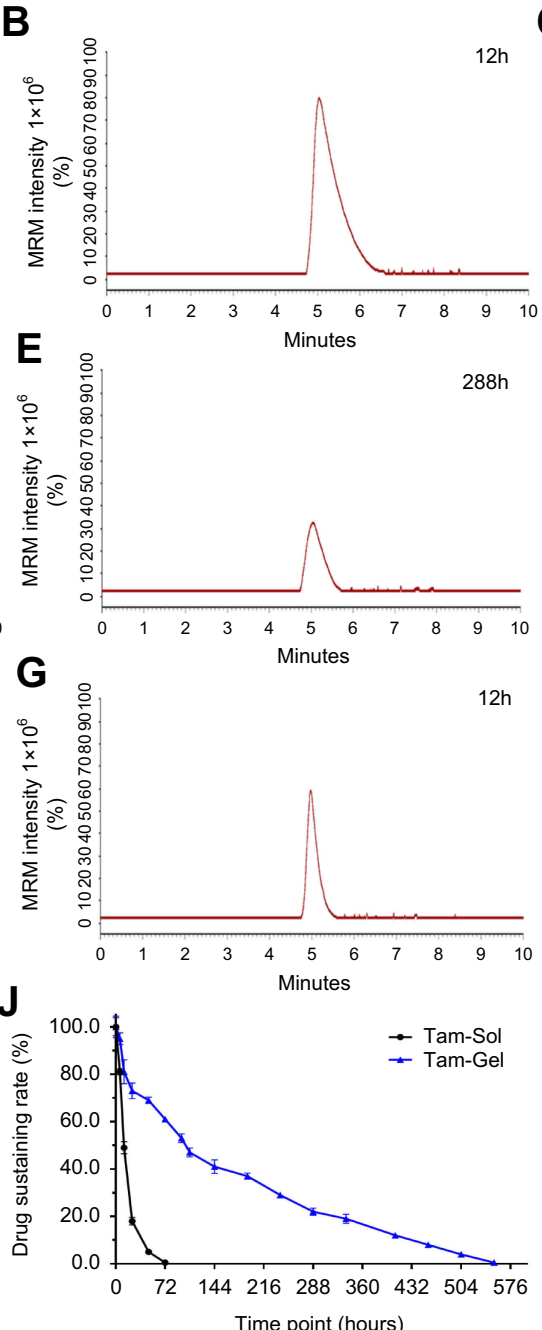

C

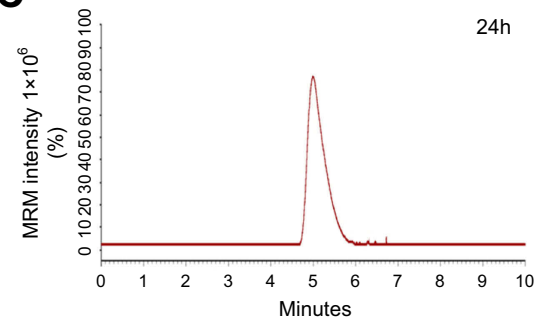

$24 \mathrm{~h}$

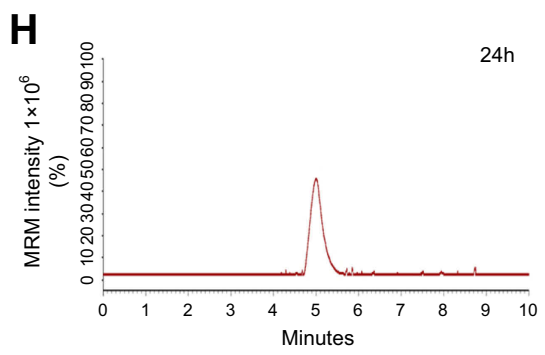

Figure 3 Preparation of Tam-Gel achieves in vivo long-term release of tamoxifen from Tam-Gel in a nude mouse subcutaneous tumor model. Tam-Gel or Tam-Sol was injected into subcutaneous tumors, and tumor tissues were collected at the indicated time-points. Then, tamoxifen sustained in the tumor tissue samples at the indicated time points was determined by LC-MS/MS experiments. For the Tam-Gel injection group, representative LC-MS/MS maps are shown at (A) 0 hours, (B) I 2 hours, (C) 24 hours, (D) 48 hours, and (E) 288 hours. For the Tam-Sol injection group, representative LC-MS/MS maps are shown at (F) 0 hours, (G) 12 hours, (H) 24 hours, and (I) 48 hours. (J) Sustained release curve of tamoxifen in Tam-Gel (mean $\pm S D$ ).

Abbreviation: LC-MS/MS, liquid chromatography-tandem mass spectrometry. 


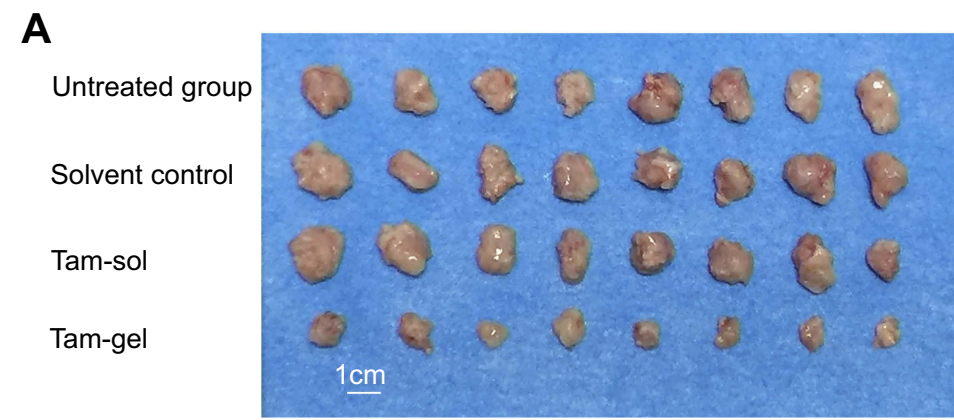

B
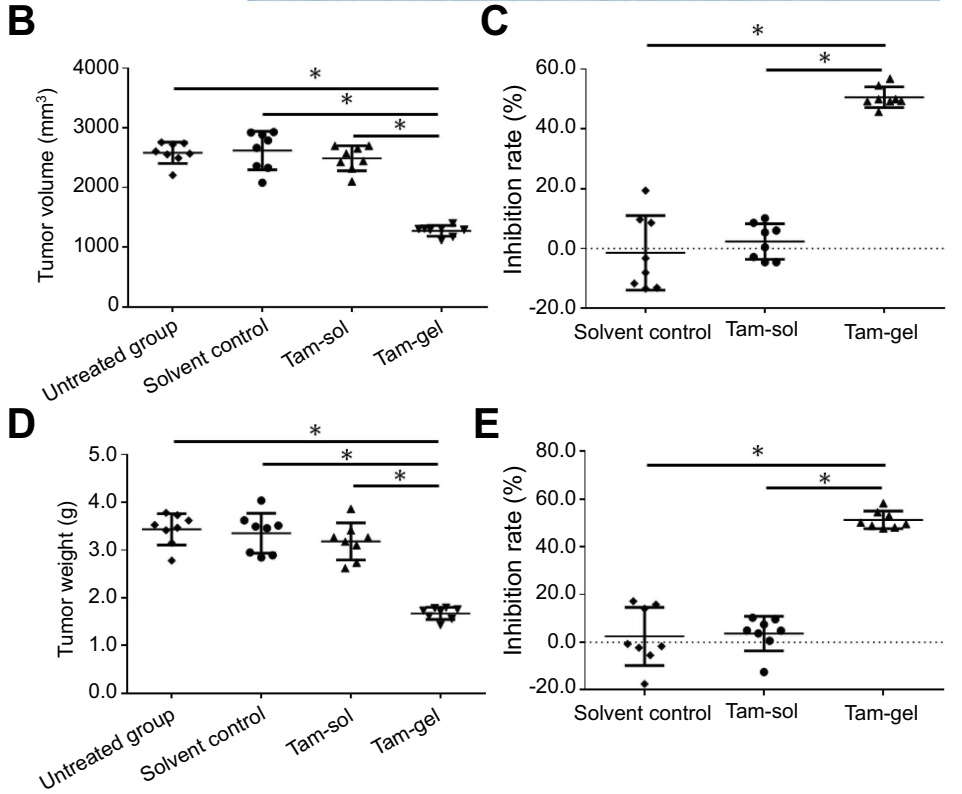

Figure 4 A single administration (one-dose administration) of Tam-Gel, but not Tam-Sol, inhibits the subcutaneous growth of MCF-7 cells in nude mice. MCF-7 cells were seeded into nude mice to form subcutaneous tumors. When tumor volumes reached $1200-1500 \mathrm{~mm}^{3}$, Tam-Gel or Tam-Sol was injected into the subcutaneous tumors. After 3-4 weeks' growth, the tumors were harvested. (A) Displays the images of the tumors. Results are shown as (B) tumor volumes, (C) inhibition rates from tumor volumes, and (D) tumor weight and (E) tumor inhibition rates from tumor weights. $* P<0.05$.

tumor tissues, tamoxifen could still be detected in the tumor tissues at the 500 hour time-point (Figure 3). Therefore, the intratumoral injection of Tam-Gel achieved the slow release and long sustainability of tamoxifen in tumor tissues formed by MCF-7 cells.

\section{Long-acting antitumor effect of a single administration of Tam-Gel in tumor tissues}

To further examine the antitumor effect of Tam-Gel on breast cancer cells, the subcutaneous model or intrahepatic model of MCF-7 was used. As shown in Figure 4, a single administration/one-dose administration of Tam-Gel, but not Tam-Sol or the solvent control, inhibited the subcutaneous growth of MCF-7 cells in nude mice.

The in vivo inhibition of activation of ER $\alpha$ by tamoxifen was examined by micro-PET screening of ${ }^{18} \mathrm{~F}$-FES absorption.
Single administration of Tam-Gel, but not Tam-Sol or the solvent control, inhibited the intrahepatic growth of MCF-7 cells in immunodeficient rats (Figures 5 and 6). The intrahepatic growth of MCF-7 cells in immunodeficient rats' livers was examined by micro-PET screening of rats after the injection of nuclear probes. First, the absorption of estrogen nuclear probes $\left({ }^{18} \mathrm{~F}-\mathrm{FES}\right)$ by MCF-7 cells was examined. As shown in Figure 5, the intratumoral injection of the solvent control did not inhibit the absorption of ${ }^{18}$ F-FES by the MCF-7 cells. A single administration of Tam-Sol by intratumoral injection inhibited the absorption of estrogen by MCF-7 cells only at day 2 but not at subsequent time-points, whereas one administration of Tam-Gel could inhibit the absorption of ${ }^{18}$ F-FES by MCF-7 cells at the indicated time-points (from day 2 to 23) for a long time.

Next, the in vivo inhibition of the MCF-7 cells' growth was examined by micro-PET screening of the ${ }^{18} \mathrm{~F}-\mathrm{FDG}$ absorption (Figures 6 and 7). The intrahepatic growth of the 


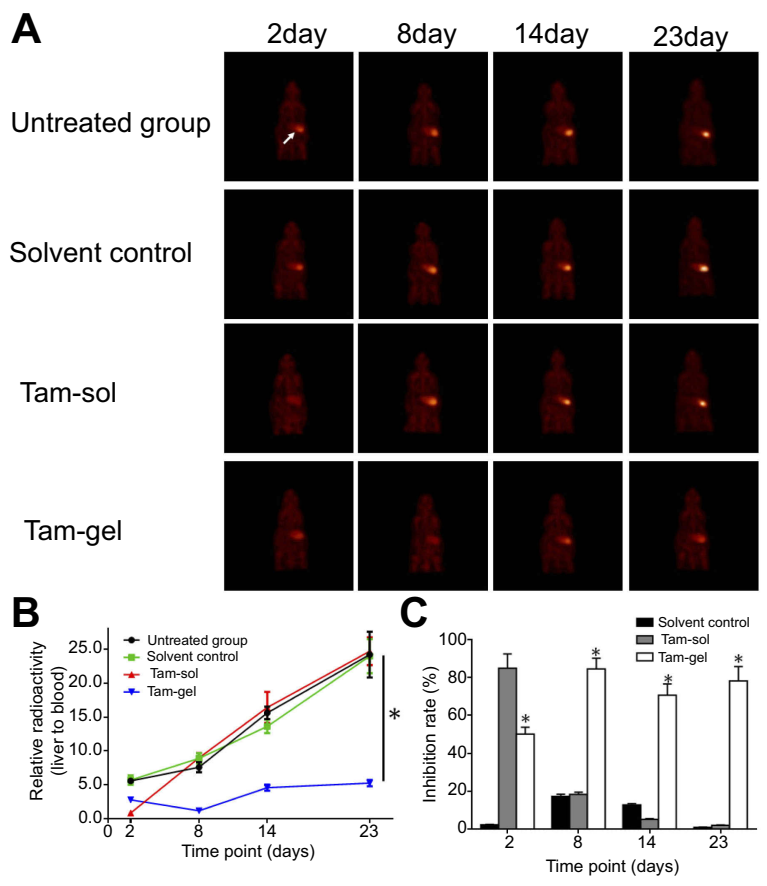

Figure 5 A single administration (one-dose administration) of Tam-Gel, but not TamSol, achieves long-acting inhibition of ${ }^{18} \mathrm{~F}$-FES absorption by MCF-7 in immunodeficient rats' liver lesions. (A) Micro-positron emission tomography images at the indicated time-points, (B) quantitative analysis results of images, and (C) inhibition rates from the quantitative analysis results of the images. $* P<0.05(\mathbf{B}) ; * P<0.05$, TamSol group vs Tam-Gel group (C).

Abbreviation: ${ }^{18} \mathrm{~F}-\mathrm{FES},{ }^{18} \mathrm{~F}$-fluoroestradiol.

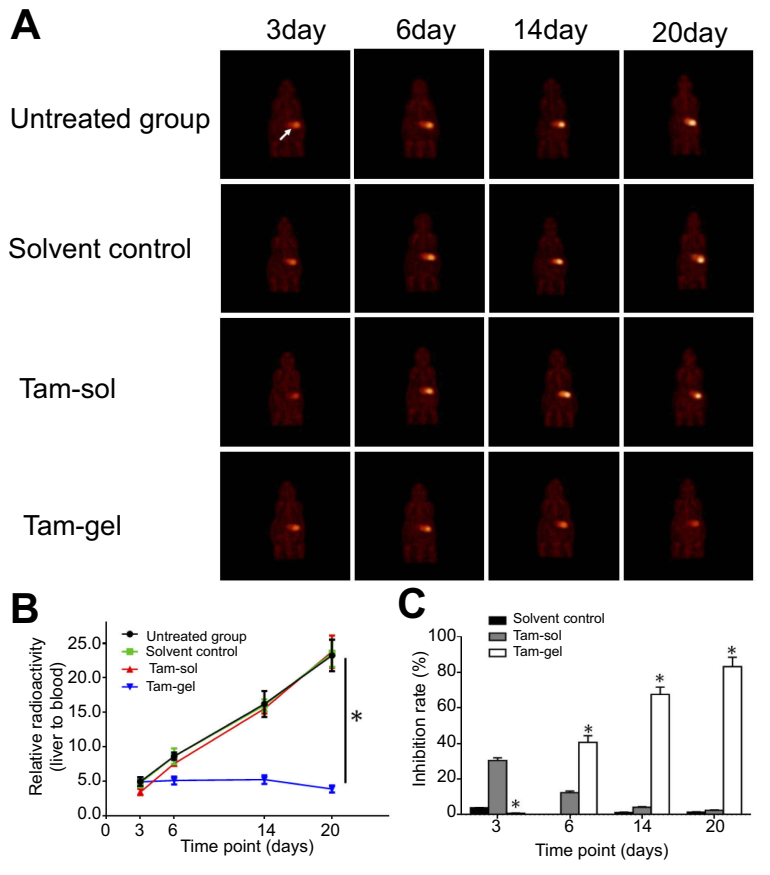

Figure 6 A single administration (one-dose administration) of Tam-Gel, but not TamSol, achieves long-acting inhibition of ${ }^{18}$ F-FDG absorption by MCF-7 in immunodeficient rats' liver lesions. (A) Micro-positron emission tomography images at the indicated time-points, (B) quantitative analysis results of images, and (C) inhibition rates from the quantitative analysis results of the images. $* P<0.05(\mathbf{B}) ; * P<0.05$, vs Tam-Sol group vs Tam-Gel group (C).

Abbreviation: ${ }^{18} \mathrm{~F}-\mathrm{FDG},{ }^{18} \mathrm{~F}$-fluorodeoxyglucose.

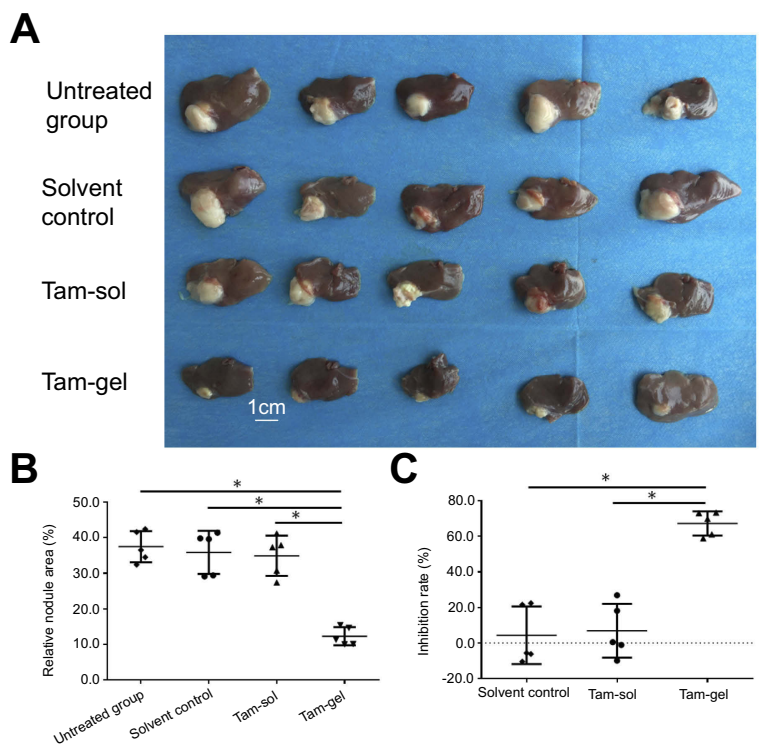

Figure 7 A single administration (one-dose administration) of Tam-Gel, but not Tam-Sol, achieves long-acting inhibition of intrahepatic growth of MCF-7 cells. (A) Photographs of intrahepatic lesions caused by MCF-7 in immunodeficient rats. (B) Quantitative results of the lesions' area by total liver area (mean \pm SD). (C) Inhibition rates calculated from the quantitative results. $* P<0.05$.

MCF-7 cells was examined by micro-PET screening of rats that received ${ }^{18} \mathrm{~F}$-FDG nuclear probes or images of rats' livers with tumor lesions formed by MCF-7 cells (Figures 6 and 7). As shown in Figure 6, a single administration of Tam-Gel, but not Tam-Sol or the solvent control, inhibited the intensity of ${ }^{18} \mathrm{~F}$-FDG nuclear probes in the liver region of rats for a long time. At day 24, rats were harvested. Images of their livers with the formed lesions are shown in Figure 7. The results indicated that only Tam-Gel, but not Tam-Sol or the solvent control, inhibited the MCF-7 tumor volumes in rats' livers. Therefore, intratumoral injection of Tam-Gel achieved the long-acting antitumor activation of tamoxifen on MCF-7 cells.

\section{Discussion}

Breast cancer is the biggest threat to female health and one of the foremost causes of death from cancers affecting females. ${ }^{36,37}$ In fact, among the three main subtypes of breast cancer, about $70 \%$ of all breast cancer patients suffer from endocrine-related/dependent breast cancer and have ER $\alpha$ positive tumors. ${ }^{38,39}$ Therefore, ER $\alpha$ is the prime target for breast cancer treatment. ${ }^{40,41}$ Antagonists of ER $\alpha$, for example, tamoxifen and fulvestrant, have been widely used to treat patients with breast cancer. ${ }^{42,43}$ In the presence of tamoxifen, $\mathrm{ER} \alpha$ binds preferentially and recruits the transcriptional corepressors, N-CoR and SMRT. ${ }^{44}$ Recruitment of these corepressors induced by tamoxifen not only decreases the expression of ER $\alpha$ 's downstream genes but also inhibits the 
proliferation of breast cancer cells. ${ }^{39,44}$ Although antitumor treatment of endocrine-dependent breast cancer has made great progress, considerably prolonging the survival of patients and improving their prognosis, current therapeutic strategies, such as oral administration of tamoxifen citrate tablets, still have many problems. These problems include: 1 ) oral administration of tamoxifen leads to a wide distribution of the drug in various organs of the human body; the effective concentration of the drug in tumor lesions is low, and the drug utilization is also insufficient; and 2) estrogen receptors not only are key regulators of normal endocrine regulation but also play significant roles in other important physiological processes, such as in osteoclasts; therefore, oral administration of estrogen receptor antagonists could interfere with the normal physiological regulation of various organs in the body while treating breast cancer, and induce side effects.

To avoid these problems, this study prepared a new pharmaceutical formulation of tamoxifen, a temperaturesensitive phase-change hydrogel of tamoxifen (Tam-Gel) , and achieved direct administration in tumor tissues. The solubility of tamoxifen is poor, and the preparation of tamoxifen as a nanoparticle not only improved its chemical properties but also accommodated large amounts of tamoxifen in small volumes. Tamoxifen nanoparticles were prepared into gels using PLGAPEG-PLGA. Tam-Gel appears as a liquid state at room temperature and undergoes a phase change to convert to a hydrogel state at body temperature. After the Tam-Gel hydrogel was injected into the tumor tissue, the drug was released by the dissolution of the gel into the tumor tissue, thereby achieving the sustained release of the drug in the tumor tissue. PLGA-PEG-PLGA is a novel organic polymer material with good safety and is fully biodegradable. ${ }^{45-47}$ It also has the chemistry of sol-gel conversion and could be used to prepare temperaturesensitive phase-change hydrogels. At body temperature, the biopolymer chains in PLGA-PEG formulations undergo stretching and cross-linking, eventually undergoing phase transformation, converting from liquid to hydrogel. ${ }^{48,49}$ The formulation has different ratios of PLGA-PEG, and its phase-transition temperatures are also different. In the present work, the phase-transition temperature of PLGA-PEG hydrogel is near body temperature. In a gel prepared with PLGA-PEG-PLGA, the amount of PLGA-PEG-PLGA is small, the mechanical strength of the gel is good, the drug is slowed down and the sustained release time is long. ${ }^{50,51}$ Therefore, the gel prepared in this study can achieve drug limitation in tumor tissues, avoiding potential toxic side effects on various organs of the body.

Current breast cancer research mainly uses breast cancer cells or patient-derived cells/tissues to establish a subcutaneous tumor model in nude mice; ${ }^{52,53}$ although the subcutaneous tumor model is widely used as a classic in vivo research method, subcutaneous tumors cannot mimic the microenvironment of human cancers, and recent studies have focused on establishing in situ tumor models (establishing a tumor tissue model in a malignant, tumor-derived organ). However, the in situ tumor models of breast cancer cells are still similar to the subcutaneous tumors. Therefore, this study used immunodeficient rats to establish the lesions caused by MCF-7 cells in the livers of the animals. Establishment of an intrahepatic tumor model in rats can mimic the liver metastasis of breast cancer and the growth of MCF-7 cells in organs. Compared with the nude mouse tumor model, the immunodeficient rats have a larger body volume. The intrahepatic tumor tissue obtained in this study can achieve intratumoral drug administration and will be widely used in the future for ultrasound-guided abdominal puncture and other precise drug-delivery methods.

In the present work, tamoxifen was used. Tamoxifen has often been administered orally to patients. There has been some research focusing on the drug-delivery system of tamoxifen nanoparticles. ${ }^{54,55}$ However, there have been no reports on the formulation of tamoxifen to maintain the drug in breast cancer. Medical image-guided interventional therapeutic strategies, such as CT-guided abdominal puncture and digital subtraction angiography-guided transcatheter arterial chemoembolization, can directly inject antitumor drugs into tumor lesions, and also achieve long-term effects of a single administration after the injection of a sustained-release preparation of the drug into tumor tissue. ${ }^{56,57}$ This strategy could not only relieve the economic burden on patients but also alleviate the side effects of the drugs. ${ }^{21}$ Currently, the choice of drugs in interventional therapeutic strategies is limited, and Tam-Gel can be accurately administered by ultrasound-guided or CT-guided percutaneous puncture. Fulvestrant (ICI182780), another antagonist of estrogen receptors, has also been widely used as first-line or second-line therapy for locally advanced or metastatic breast cancer. ${ }^{58,59}$ Fulvestrant is also the foremost therapeutic choice for post-menopausal patients with breast cancer. ${ }^{60,61}$ In the future, we will try to establish various new pharmaceutical formulations of fulvestrant to achieve more effective antitumor treatments for breast cancer. 
Moreover, there are other strategies to prepare controlledrelease or slow-release systems. ${ }^{62-64}$ The drug loading amount in the present work is almost $2 \mathrm{mg} / \mathrm{mL}$. It will be valuable to develop novel controlled-release or slow-release systems for drugs by other strategies.

\section{Conclusion}

In this study, a novel slow-release system was successfully prepared that facilitates the long-term release of tamoxifen in breast cancer tissues and achieves an antitumor effect in the long term.

\section{Acknowledgment}

We thank Dr Xiaojie Xu, Department of Medical Molecular Biology, Beijing Institute of Biotechnology, Beijing, People's Republic of China for her kind advice.

\section{Author contributions}

All authors made substantial contributions to the design and conception; and the acquisition, analysis or interpretation of data. All authors took part in either drafting or revising the manuscript. All authors gave final approval of the version to be published; and agree to be accountable for all aspects of the work in ensuring that questions related to the accuracy or integrity of any part of the work are appropriately investigated and resolved.

\section{Disclosure}

The authors report no conflicts of interest in this work.

\section{References}

1. Vaidya JS, Bulsara M, Wenz F, Tobias JS, Joseph D, Baum M. Targeted radiotherapy for early breast cancer. Lancet. 2018;391:26-27. doi:10.1016/S0140-6736(17)33316-0

2. Allemani C, Matsuda T, Di Carlo V, et al.; CONCORD Working Group. Global surveillance of trends in cancer survival 2000-14 (CONCORD-3): analysis of individual records for 37513025 patients diagnosed with one of 18 cancers from 322 populationbased registries in 71 countries. Lancet. 2018;391:1023-1075. doi:10.1016/S0140-6736(17)33326-3

3. Rimawi M, Ferrero JM, de la Haba-Rodriguez J, et al.; PERTAIN Study Group. First-line trastuzumab plus an aromatase inhibitor, with or without pertuzumab, in human epidermal growth factor receptor 2-positive and hormone receptor-positive metastatic or locally advanced breast cancer (PERTAIN): a randomized, open-label Phase II trial. J Clin Oncol. 2018;36:2826-2835. doi:10.1200/JCO.2017.76.7863

4. Razavi P, Chang MT, Xu G, et al. The genomic landscape of endocrine-resistant advanced breast cancers. Cancer Cell. 2018;34:427-438.e6. doi:10.1016/j.ccell.2018.08.008

5. Zhang W, Yu W, Cai G, et al. A new synthetic derivative of cryptotanshinone KYZ3 as STAT3 inhibitor for triple-negative breast cancer therapy. Cell Death Dis. 2018;9:1098. doi:10.1038/s41419-018-1111-y
6. Meng D, Lei M, Han Y, et al. MicroRNA-645 targets urokinase plasminogen activator and decreases the invasive growth of MDA-MB-231 triple-negative breast cancer cells. Onco Targets Ther. 2018;11:7733-7743. doi:10.2147/OTT.S187221

7. Shah N, Mohammad AS, Saralkar P, et al. Investigational chemotherapy and novel pharmacokinetic mechanisms for the treatment of breast cancer brain metastases. Pharmacol Res. 2018;132:47-68. doi:10.1016/j. phrs.2018.03.021

8. Zhu Z, Li Y, Yang X, Pan W, Pan H. The reversion of anti-cancer drug antagonism of tamoxifen and docetaxel by the hyaluronic acid-decorated polymeric nanoparticles. Pharmacol Res. 2017;126:84-96. doi:10.1016/j. phrs.2017.07.011

9. Goetz MP, Suman VJ, Reid JM, et al. First-in-human Phase I study of the tamoxifen metabolite Z-endoxifen in women with endocrine-refractory metastatic breast cancer. J Clin Oncol. 2017;35:3391-3400. doi:10.1200/ JCO.2017.73.3246

10. Davies C, Pan H, Peto R. 10 vs 5 years of adjuvant tamoxifen: exclusion of 1/402 centres in ATLAS. Lancet. 2017;389:1884 doi:10.1016/S0140-6736(17)31003-6

11. Rocca A, Maltoni R, Bravaccini S, Donati C, Andreis D. Clinical utility of fulvestrant in the treatment of breast cancer: a report on the emerging clinical evidence. Cancer Manag Res. 2018;10:3083-3099. doi:10.2147/CMAR.S137772

12. Li J, Li B, Jiang Q, et al. Do genetic polymorphisms of the vitamin $\mathrm{D}$ receptor contribute to breast/ovarian cancer? A systematic review and network meta-analysis. Gene. 2018;677:211-227. doi:10.1016/j.gene.2018.07.070

13. Khan MM. Translational significance of selective estrogen receptor modulators in psychiatric disorders. Int J Endocrinol. 2018;2018:9516592. doi:10.1155/2018/1528437

14. Sharma D, Kumar S, Narasimhan B. Estrogen alpha receptor antagonists for the treatment of breast cancer: a review. Chem Cent J. 2018;12:107. doi:10.1186/s13065-018-0472-8

15. Meitzen J, Meisel RL, Mermelstein PG. Sex differences and the effects of estradiol on striatal function. Curr Opin Behav Sci. 2018;23:42-48. doi:10.1016/j.cobeha.2018.03.007

16. Xie H, Tian S, Yu H, et al. A new apatinib microcrystal formulation enhances the effect of radiofrequency ablation treatment on hepatocellular carcinoma. Onco Targets Ther. 2018;11:3257-3265. doi:10.2147/OTT.S165000

17. Wang Y, Tang G. A novel long-sustaining system of apatinib to long-termly inhibit the proliferation of hepatocellular carcinoma cells. Onco Targets Ther. 2018;11:8529-8541. doi:10.2147/OTT. S188209

18. De Placido S, Gallo C, De Laurentiis M, et al. Adjuvant anastrozole versus exemestane versus letrozole, upfront or after 2 years of tamoxifen, in endocrine-sensitive breast cancer (FATA-GIM3): a randomised, phase 3 trial. Lancet Oncol. 2018;19:474-485. doi:10.1016/S1470-2045(18)30116-5

19. Dehghani F, Farhadian N, Golmohammadzadeh S, Biriaee A, Ebrahimi M, Karimi M. Preparation, characterization and in-vivo evaluation of microemulsions containing tamoxifen citrate anti-cancer drug. Eur J Pharm Sci. 2017;96:479-489. doi:10.1016/j.ejps.2016.09.033

20. Peris-Vicente J, Carda-Broch S, Esteve-Romero J. Quantification of tamoxifen in pharmaceutical formulations using micellar liquid chromatography. Anal Sci. 2014;30:925-930.

21. Park CG, Hartl CA, Schmid D, Carmona EM, Kim HJ, Goldberg MS. Extended release of perioperative immunotherapy prevents tumor recurrence and eliminates metastases. Sci Transl Med. 2018;10:pii: eaar1916. doi:10.1126/scitranslmed.aao4496

22. Hyun MH, Lee YS, Kim JH, et al. Hepatic resection compared to chemoembolization in intermediate- to advanced-stage hepatocellular carcinoma: a meta-analysis of high-quality studies. Hepatology. 2018;68:977-993. doi:10.1002/hep.29883 
23. Lo GH. The use of steroid for transcatheter arterial chemoembolization: to relieve symptoms or to mask adverse events? Hepatology. 2018;68:1207. doi:10.1002/hep.30112

24. Majumder P, Baxa U, Walsh STR, Schneider JP. Design of a multicompartment hydrogel that facilitates time-resolved delivery of combination therapy and synergized killing of glioblastoma. Angew Chem Int Ed Engl. 2018;57:15040-15044. doi:10.1002/ anie. 201806483

25. Li J, Mooney DJ. Designing hydrogels for controlled drug delivery. Nat Rev Mater. 2016;1:pii: 16071. doi:10.1038/natrevmats.2016.71

26. Zhang F, Feng F, Yang P, et al. Four-and-a-half-LIM protein 1 downregulates estrogen receptor $\alpha$ activity through repression of AKT phosphorylation in human breast cancer cell. Int $J$ Biochem Cell Biol. 2012;44:320-326. doi:10.1016/j.biocel.2011.11.002

27. Johänning J, Heinkele G, Precht JC, et al. Highly sensitive simultaneous quantification of estrogenic tamoxifen metabolites and steroid hormones by LC-MS/MS. Anal Bioanal Chem. 2015;407:7497-7502. doi:10.1007/s00216-015-8907-8

28. Rama Raju KS, Taneja I, Singh SP, et al. Simultaneous determination of centchroman and tamoxifen along with their metabolites in rat plasma using LC-MS/MS. Bioanalysis. 2015;7:967-979. doi:10.4155/bio.14.253

29. An L, Li DD, Chu HX, et al. Terfenadine combined with epirubicin impedes the chemo-resistant human non-small cell lung cancer both in vitro and in vivo through EMT and notch reversal. Pharmacol Res. 2017;124:105-115. doi:10.1016/j.phrs.2017.07.021

30. Ji Q, Xu X, Li L, et al. miR-216a inhibits osteosarcoma cell proliferation, invasion and metastasis by targeting CDK14. Cell Death Dis. 2017;8:e3103. doi:10.1038/cddis.2017.518

31. Xu X, Fan Z, Kang L, et al. Hepatitis B virus X protein represses miRNA-148a to enhance tumorigenesis. $J$ Clin Invest. 2013;123:630-645. doi:10.1172/JCI64265

32. Feng F, Jiang Q, Cao S, et al. Pregnane X receptor mediates sorafenib resistance in advanced hepatocellular carcinoma. Biochim Biophys Acta Gen Subj. 2018;1862:1017-1030. doi:10.1016/j.bbagen.2018.01.011

33. Li L, Liang Y, Kang L, et al. Transcriptional regulation of the warburg effect in cancer by SIX1. Cancer Cell. 2018;33:368-385. e7. doi:10.1016/j.ccell.2018.01.010

34. Li J, Zhao J, Wang H, et al. MicroRNA-140-3p enhances the sensitivity of hepatocellular carcinoma cells to sorafenib by targeting pregnenolone X receptor. Onco Targets Ther. 2018;11:5885-5894. doi:10.2147/OTT.S179509

35. Shao Z, Li Y, Dai W, et al. ETS-1 induces sorafenib-resistance in hepatocellular carcinoma cells via regulating transcription factor activity of PXR. Pharmacol Res. 2018;135:188-200. doi:10.1016/j. phrs.2018.08.003

36. Bray F, Ferlay J, Soerjomataram I, Siegel RL, Torre LA, Jemal A. Global cancer statistics 2018: GLOBOCAN estimates of incidence and mortality worldwide for 36 cancers in 185 countries. CA Cancer J Clin. 2018;68:394-424. doi:10.3322/caac.21492

37. Fan W, Xie J, Xia J, et al. RUVBL1-ITFG1 interaction is required for collective invasion in breast cancer. Biochim Biophys Acta Gen Subj. 2017;1861:1788-1800. doi:10.1016/j.bbagen.2017.03.016

38. Biava PM, Nicolini A, Ferrari P, Carpi A, Sell S. A systemic approach to cancer treatment: tumor cell reprogramming focused on endocrine-related cancers. Curr Med Chem. 2014;21:1072-1081.

39. Cui J, Germer K, Wu T, et al. Cross-talk between HER2 and MED1 regulates tamoxifen resistance of human breast cancer cells. Cancer Res. 2012;72:5625-5634. doi:10.1158/0008-5472.CAN-12-1305

40. Johmura Y, Maeda I, Suzuki N, et al. Fbxo22-mediated KDM4B degradation determines selective estrogen receptor modulator activity in breast cancer. J Clin Invest. 2018;pii: 121679. doi:10.1172/ JCI121679

41. Wang L, Zhao L, Jia X, et al. Aminophenols increase proliferation of thyroid tumor cells by inducing the transcription factor activity of estrogen receptor $\alpha$. Biomed Pharmacother. 2018;109:621-628. doi:10.1016/j.biopha.2018.10.168
42. Cun J, Yang Q. Bioinformatics-based interaction analysis of miR-92a-3p and key genes in tamoxifen-resistant breast cancer cells. Biomed Pharmacother. 2018;107:117-128. doi:10.1016/j. biopha.2018.07.158

43. Kornblum N, Zhao F, Manola J, et al. Randomized Phase II trial of fulvestrant plus everolimus or placebo in postmenopausal women with hormone receptor-positive, human epidermal growth factor receptor 2-negative metastatic breast cancer resistant to aromatase Inhibitor therapy: results of PrE0102. J Clin Oncol. 2018;36:1556-1563. doi:10.1200/ JCO.2017.76.9331

44. Peterson TJ, Karmakar S, Pace MC, Gao T, Smith CL. The silencing mediator of retinoic acid and thyroid hormone receptor (SMRT) corepressor is required for full estrogen receptor alpha transcriptional activity. Mol Cell Biol. 2007;27:5933-5948. doi:10.1128/ MCB.00237-07

45. El-Zaafarany GM, Soliman ME, Mansour S, et al. A tailored thermosensitive PLGA-PEG-PLGA/emulsomes composite for enhanced oxcarbazepine brain delivery via the nasal route. Pharmaceutics. 2018;10:pii: E217. doi:10.3390/pharmaceutics10040217

46. Pan A, Wang Z, Chen B, et al. Localized co-delivery of collagenase and trastuzumab by thermosensitive hydrogels for enhanced antitumor efficacy in human breast xenograft. Drug Deliv. 2018;25:1495-1503. doi:10.1080/10717544.2018.1474971

47. Shi Z, Li SK, Charoenputtakun P, Liu CY, Jasinski D, Guo P. RNA nanoparticle distribution and clearance in the eye after subconjunctival injection with and without thermosensitive hydrogels. J Control Release. 2018;270:14-22. doi:10.1016/j.jconrel.2017.11.028

48. GuhaSarkar S, Banerjee R. Intravesical drug delivery: challenges, current status, opportunities and novel strategies. $J$ Control Release. 2010;148:147-159. doi:10.1016/j.jconrel.2010.08.031

49. Mukerjee A, Ranjan AP, Vishwanatha JK. Combinatorial nanoparticles for cancer diagnosis and therapy. Curr Med Chem. 2012;19:3714-3721.

50. Santoveña A, Monzón C, Alvarez-Lorenzo C, et al. Structureperformance relationships of temperature-responsive PLGA-PEGPLGA gels for sustained release of bone morphogenetic protein-2. J Pharm Sci. 2017;106:3353-3362. doi:10.1016/j.xphs.2017.07.007

51. Dimchevska S, Geskovski N, Koliqi R, et al. Efficacy assessment of self-assembled PLGA-PEG-PLGA nanoparticles: correlation of nano-bio interface interactions, biodistribution, internalization and gene expression studies. Int $J$ Pharm. 2017;533:389-401. doi:10.1016/j. ijpharm.2017.05.054

52. Feng Z, Xia Y, Gao T, et al. The antipsychotic agent trifluoperazine hydrochloride suppresses triple-negative breast cancer tumor growth and brain metastasis by inducing G0/G1 arrest and apoptosis. Cell Death Dis. 2018;9:1006. doi:10.1038/s41419-018-1111-y

53. Zhou EY, Knox HJ, Reinhardt CJ, Partipilo G, Nilges MJ, Chan J. Near-infrared photoactivatable nitric oxide donors with integrated photoacoustic monitoring. J Am Chem Soc. 2018;140:11686-11697. doi:10.1021/jacs.8b05514

54. Patel SH, O'Hara L, Atanassova N, et al. Low-dose tamoxifen treatment in juvenile males has long-term adverse effects on the reproductive system: implications for inducible transgenics. Sci Rep. 2017;7:8991. doi:10.1038/s41598-017-09016-4

55. Dhaundiyal A, Jena SK, Samal SK, Sonvane B, Chand M, Sangamwar AT. Alpha-lipoic acid-stearylamine conjugate-based solid lipid nanoparticles for tamoxifen delivery: formulation, optimization, in-vivo pharmacokinetic and hepatotoxicity study. J Pharm Pharmacol. 2016;68:1535-1550. doi:10.1111/jphp.12644

56. Xie H, Yu H, Tian S, et al. What is the best combination treatment with transarterial chemoembolization of unresectable hepatocellular carcinoma? a systematic review and network meta-analysis. Oncotarget. 2017;8:100508-100523. doi:10.18632/oncotarget.20119

57. Feng F, Jiang Q, Jia H, et al. Which is the best combination of TACE and Sorafenib for advanced hepatocellular carcinoma treatment? A systematic review and network meta-analysis. Pharmacol Res. 2018;135:89-101. doi:10.1016/j.phrs.2018.06.021 
58. Zhang $\mathrm{K}$, Hong $\mathrm{R}, \mathrm{Xu} \mathrm{F}$, et al. Clinical value of circulating ESR1 mutations for patients with metastatic breast cancer: a meta-analysis Cancer Manag Res. 2018;10:2573-2580. doi:10.2147/CMAR.S173193

59. Du Y, Li N, Jiao X, Li K, Yan S. The predictive ability of plasma ESR1 mutations for the efficacy of endocrine therapy in hormone-receptor-positive advanced breast cancer. Onco Targets Ther. 2018;11:6023-6029. doi:10.2147/OTT.S171465

60. Rusz O, Kószó R, Dobi Á, et al. Clinical benefit of fulvestrant monotherapy in the multimodal treatment of hormone receptor and HER2 positive advanced breast cancer: a case series. Onco Targets Ther. 2018;11:5459-5463. doi:10.2147/OTT.S170736

61. Zhang T, Feng F, Zhao W, et al. Effect of first-line endocrine therapy in patients with hormone-sensitive advanced breast cancer: a network meta-analysis. Onco Targets Ther. 2018;11:2647-2656. doi:10.2147/ OTT.S165681
62. Nosrati H, Salehiabar M, Davaran S, Danafar H, Manjili HK. Methotrexate-conjugated L-lysine coated iron oxide magnetic nanoparticles for inhibition of MCF-7 breast cancer cells. Drug Dev Ind Pharm. 2017;27:1-9.

63. Nosrati H, Adinehvand R, Manjili HK, Rostamizadeh K, Danafar H. Synthesis, characterization, and kinetic release study of methotrexate loaded mPEG-PCL polymersomes for inhibition of MCF-7 breast cancer cell line. Pharm Dev Technol. 2019;24:89-98. doi:10.1080/ 10837450.2018.1425433

64. Nosrati H, Rashidi N, Danafar H, Manjili HK. Anticancer activity of tamoxifen loaded tyrosine decorated biocompatible $\mathrm{Fe} 3 \mathrm{O} 4$ magnetic nanoparticles against breast cancer cell lines. $J$ Inorg Organomet Polym Mater. 2018;28:1178-1186. doi:10.1007/s10904-017-0758-7 


\section{Supplementary materials}

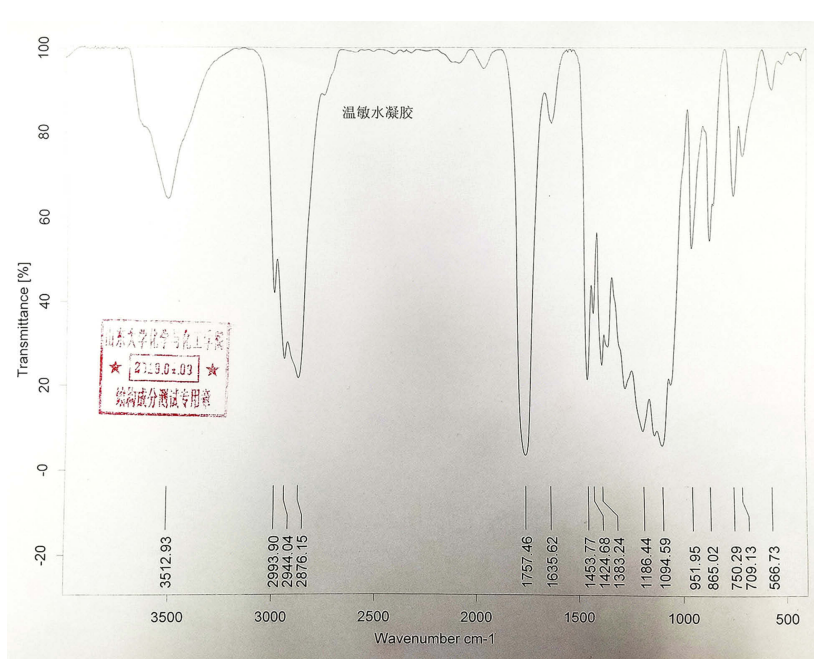

Figure SI The infrared detection of PLGA-PEG.

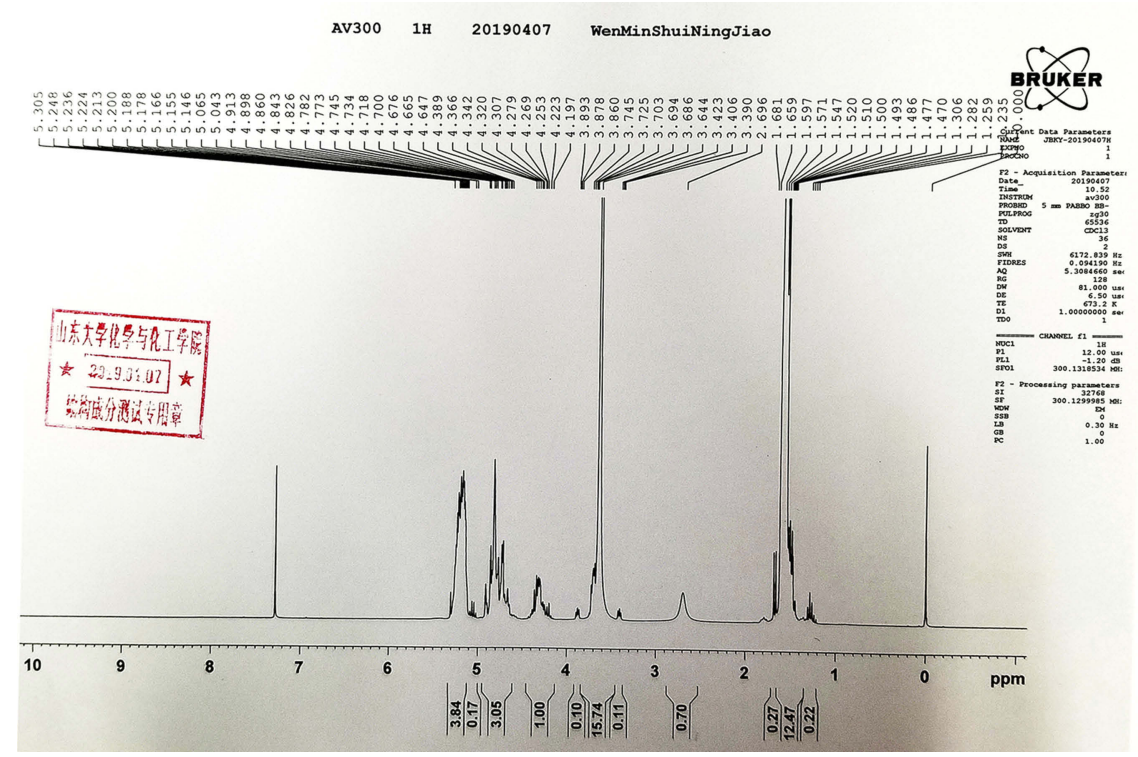

Figure S2 >Nuclear magnetic detection of PLGA-PEG.

Table SI Weights of tumor tissues seeded into rats' livers

\begin{tabular}{|l|l|l|l|l|}
\hline \multirow{2}{*}{ Animal } & Untreated group & Solvent control & \multicolumn{2}{l|}{ Tam-Sol } \\
\cline { 2 - 5 } & Weight of tumor tissues seeded (mg) & \multicolumn{2}{|l|}{ Tam-Gel } \\
\hline No. 1 & 109.7 & 115.4 & 120.6 & 115.8 \\
No. 2 & 111.9 & 117.1 & 102.3 & 109.8 \\
No. 3 & 106.7 & 114.3 & 120.1 & 106.6 \\
No. 4 & 104.7 & 111.6 & 110.5 & 108.8 \\
No. 5 & 116.1 & 109.2 & 110.3 & 107.4 \\
\hline
\end{tabular}




\section{Publish your work in this journal}

OncoTargets and Therapy is an international, peer-reviewed, open access journal focusing on the pathological basis of all cancers, potential targets for therapy and treatment protocols employed to improve the management of cancer patients. The journal also focuses on the impact of management programs and new therapeutic agents and protocols on patient perspectives such as quality of life, adherence and satisfaction. The manuscript management system is completely online and includes a very quick and fair peer-review system, which is all easy to use. Visit http://www.dovepress.com/ testimonials.php to read real quotes from published authors.

Submit your manuscript here: https://www.dovepress.com/oncotargets-and-therapy-journal 